Revue européenne des migrations internationales

vol. $27-n^{\circ} 1 \mid 2011$

La construction de l'altérité dans l'espace noir atlantique : Etats-Unis - France - Caraïbes - Amérique latine

\title{
Catherine GOUSSEFF, L'exil russe. La fabrique du réfugié apatride (1920-1939)
}

\section{Bénédicte Michalon}

\section{OpenEdition \\ Journals}

Édition électronique

URL : https://journals.openedition.org/remi/5413

DOI : $10.4000 /$ remi.5413

ISSN : $1777-5418$

Éditeur

Université de Poitiers

Édition imprimée

Date de publication : 1 juin 2011

Pagination : 177-180

ISBN : 979-10-90426-00-9

ISSN : 0765-0752

Référence électronique

Bénédicte Michalon, « Catherine GOUSSEFF, L'exil russe. La fabrique du réfugié apatride (1920-1939) », Revue européenne des migrations internationales [En ligne], vol. $27-n^{\circ} 1$ | 2011, mis en ligne le 30 août 2011, consulté le 14 avril 2022. URL : http://journals.openedition.org/remi/5413 ; DOI : https://doi.org/ 10.4000/remi.5413

Ce document a été généré automatiquement le 14 avril 2022.

(C) Université de Poitiers 


\title{
Catherine GOUSSEFF, L'exil russe. La fabrique du réfugié apatride (1920-1939)
}

\author{
Bénédicte Michalon
}

\section{RÉFÉRENCE}

Catherine GOUSSEFF, L'exil russe. La fabrique du réfugié apatride (1920-1939), Paris, CNRS

Éditions, 2008, 335 p., ISBN : 978-2-271-06621-3.

1 L'exil russe de Catherine Gousseff est un ouvrage incontournable pour qui s'intéresse aux migrations russes et à leur histoire. Comme le sous-titre l'indique, c'est également une référence pour la connaissance du droit d'asile et du statut de réfugié. Au travers d'une histoire sociale de l'immigration russe en France pendant l'entre-deux guerres, Catherine Gousseff déplace le regard historiographique, venu bien souvent «de l'intérieur ». Elle revisite de nombreux mythes qui prévalent sur cette immigration et livre quantité d'informations inédites. Ce faisant, l'auteure inscrit l'exil des Russes dans l'histoire française et européenne des migrations et nous livre une analyse de l'émergence de la question des réfugiés sur la scène internationale et de son institutionnalisation par les États européens. C'est finalement le rôle central joué par ces émigrés dans l'élaboration et la mise en œuvre d'une rationalité gestionnaire de l'exil et de l'asile qui est mis en lumière.

2 Les parcours des émigrants sont d'abord appréhendés à l'échelle de leur dispersion, celle de l'Europe et des fins d'empires des lendemains de la Première Guerre mondiale. L'ouvrage restitue la diversité des trajectoires sociales, géographiques et politiques, et tord ainsi le cou à de nombreux stéréotypes sur ce flux. Les émigrés de l'entre-deux guerres appartenaient en effet aux classes les plus diverses de la société russe et constituaient un ensemble hétérogène. Les anciens militaires de l'armée tsariste sont les plus connus d'entre eux; ils étaient néanmoins accompagnés de représentants de l'élite administrative de l'empire, de notables urbains et de membres de ce que l'auteure qualifie de "nouvelles classes moyennes ». Les immigrants de milieux plus 
défavorisés, venus de la paysannerie principalement, représentaient le tiers des arrivants. C'est donc une société complexe qui a immigré. Les réfugiés provenaient des régions occidentales de la Russie : $60 \%$ d'entre eux étaient originaires du Sud, des villes d'Ukraine et des provinces cosaques. Un cinquième venait du nord de la Russie, et plus particulièrement des capitales. Les zones marquées par les conflits armés étaient donc les principales pourvoyeuses d'exilés, ce qui témoignait du rôle joué par la guerre dans les motivations de l'émigration. De manière plus générale, le départ des Russes a été le premier des déplacements massifs de population consécutifs au redécoupage des frontières de l'après-guerre. Catherine Gousseff analyse dans le détail les parcours migratoires : les émigrants sont principalement sortis par le sud de la Russie et le Bosphore et sont parvenus en France par voie maritime. Deux voies continentales ont aussi existé, l'une par la Pologne, l'autre par le Bosphore et les Balkans. La présence simultanée, dans ces pays, de réfugiés et de résidents russes restés sur place après la modification de certains tracés frontaliers et, de façon parallèle, les mouvements d'aller et retour entre les États d'accueil et la Russie puis l'Union soviétique (fuite, expulsions, départs liés à la famine de 1921, retours en URSS) ont complexifié la donne et rendu les décomptes des émigrants difficiles. Alors que leur nombre était évalué selon les sources entre 700000 et 2 millions de personnes pour l'Europe, l'auteure avance qu'ils ont été près de 80000 à rejoindre la France au début des années 1920 (et non 400 000, comme cela était fréquemment défendu).

Le caractère massif de cette émigration a entraîné une forte mobilisation des États européens. La création en 1920 du Bureau d'information et d'enregistrement des réfugiés russes, puis en 1921 du Haut Commissariat aux réfugiés russes a marqué le début d'une prise en charge rationalisée des exilés. Au tout début des années 1920, le Haut Commissariat défendait aussi une politique d'aide au retour en URSS, rendue possible par la reconnaissance internationale de l'Union en 1922-1924 et soutenue par le gouvernement soviétique lui-même. Mais cette politique a été abandonnée au bout de quelques années; elle avait été reçue de façon mitigée par les exilés, qui y voyaient un mode de reconnaissance explicite de l'État soviétique dont beaucoup d'entre eux souhaitaient, en réalité, la disparition. L'ouvrage démontre donc que les premiers pas de la catégorisation juridique, de l'institutionnalisation et de la gestion internationale du réfugié découlent du dispositif d'accueil des exilés russes au début des années 1920.

La France s'est particulièrement impliquée dans la défense et l'encadrement de ce mouvement migratoire. Cela contribue à expliquer l'ampleur de l'immigration russe sur le territoire français, dont l'analyse fait l'objet de la deuxième partie de l'ouvrage. L'auteure lève le voile sur les raisons de cet engagement et sur les répercussions qu'il a eues sur l'installation des réfugiés dans le pays. Sous couvert de la tradition d'accueil républicaine, deux logiques bien distinctes l'une de l'autre ont en réalité motivé l'ouverture des autorités françaises à l'immigration russe : la volonté d'accueillir les émigrés anti-bolchéviques et ainsi d'affirmer l'opposition du gouvernement français au régime soviétique d'une part ; la nécessité de répondre aux besoins en main-d'œuvre de l'économie française de l'après-guerre d'autre part.

5 L'immigration russe des années 1920 et 1930 ne peut se comprendre qu'en étant réintégrée dans l'ensemble des migrations qui touchaient alors la France, et c'est un des apports importants de la recherche de Catherine Gousseff. Au cours de ces deux décennies, l'évolution de l'immigration russe a été tout à fait similaire à celle de l'ensemble des arrivées dans le pays, avec des entrées relativement faibles entre 1918 et 
1922, un pic très net entre 1923 et 1926, un dernier regain entre 1928 et 1930, et un ralentissement au cours des années suivantes. Cette courbe irrégulière traduit le traitement évolutif de l'asile par les dirigeants français. Lorsque l'économie nationale avait besoin de main-d'œuvre, les autorités attribuaient le titre de réfugié à tous les immigrants russes, y compris quand ceux-ci réémigraient des Balkans ou de Pologne du fait des difficultés économiques qu'ils y rencontraient. En revanche, lorsque le marché de l'emploi ralentissait, le gouvernement français soumettait l'asile aux mêmes restrictions que les entrées de main-d'œuvre. Les Russes, apatrides (en 1921 l'URSS a déchu les émigrés de leur nationalité), n'ont pas bénéficié d'un traitement particulier ; ils n'ont pas été protégés contre les expulsions et ont été soumis aux mêmes législations que les autres travailleurs étrangers. De façon plus précise, bon nombre des réfugiés ont été sélectionnés et accueillis selon les mêmes modalités que les autres catégories d'immigrants : l'arrivée des réfugiés russes faisait partie intégrante d'une politique générale d'immigration en France.

6 L'originalité du traitement institutionnel de cette migration repose dans une combinaison inédite de l'asile et du recrutement de main-d'œuvre, exposée de façon détaillée. Les Russes ont fait l'objet d'une politique de recrutement et de placement qui répondait aux nécessités économiques du pays et qui a servi à l'État français pour canaliser l'accueil des réfugiés - tout comme les entrées d'autres populations étrangères. En collaboration avec le Bureau International du Travail, des organismes de recrutement privés et des organisations de défense des émigrés russes, l'État a procédé à une sélection des exilés, à leur répartition dans certains secteurs d'activité et à leur diffusion sur le territoire national.

7 L'ouvrage aborde l'un après l'autre ces aspects de la vie des Russes en exil. Leur forte présence à Paris notamment fait partie des images qui occultent d'autres facettes de leur histoire en France. Ils connaissaient, certes une concentration dans la capitale supérieure à celle d'autres populations étrangères, certes ils se regroupaient dans quelques quartiers parisiens et dans certaines activités (industrie automobile, taxis, artisanat, professions libérales), ce qui leur conférait une visibilité indéniable, cependant la politique de main-d'œuvre de l'État français a amené une partie importante d'entre eux en province; l'analyse de ces «trajectoires méconnues » est l'un des aspects les plus novateurs de la recherche de Catherine Gousseff. Elle démontre qu'ils ont été dispersés sur l'ensemble du territoire, davantage que d'autres populations étrangères, et ont été particulièrement présents dans quelques régions industrielles. À la différence de mouvements de travailleurs antérieurs, les Russes se sont dirigés vers la Moselle, le Loiret, le Rhône, des régions industrielles « jeunes » développées après la guerre. Ils y sont arrivés en même temps que d'autres travailleurs et se sont concentrés autour de leurs bassins d'emploi. Cette organisation géographique calée sur la carte industrielle témoignait de leur forte assimilation à l'ensemble de la population étrangère : ils ont suivi les mêmes trajectoires que les autres immigrants placés. Il faut néanmoins mentionner deux exceptions. La Côte d'Azur, deuxième région d'installation, répondait à des logiques différentes, plus autonomes, transitoires et saisonnières. Enfin, les Russes ont été placés dans les campagnes de l'Ouest et du SudOuest. Ils ont été installés comme métayers de manière à ce qu'ils se fixent dans leurs régions d'installation (Gers, Haute-Garonne, Lot-et-Garonne). Lorsqu'à la veille de la Seconde Guerre mondiale de nombreux Russes ont quitté les régions industrielles fortement affectées par le chômage, les implantations agricoles ont à l'inverse exercé une attractivité nette sur les travailleurs. Le dispositif de placement agricole résultait 
d'une collaboration étroite entre les pouvoirs publics, le BIT et le Zemgor (organisme issu des administrations locales de l'empire russe et animé par d'anciens administrateurs en exil), chargé de sélectionner des émigrés dans les Balkans et en Europe centrale. Si cette politique de placement agricole destinée aux Russes était marginale, elle se caractérisait par son originalité ; elle n'a laissé que peu de traces et est restée méconnue jusqu'à ce que Catherine Gousseff s'en empare.

Le système a fonctionné grâce à la très forte implication des représentants de l'émigration dans le placement des co-nationaux, autre singularité de l'immigration russe. La mobilisation de l'élite russe en exil est au cœur de la troisième partie de l'ouvrage, qui revisite l'histoire de l'asile politique et de la construction de la catégorie juridique du « réfugié ». L'émigration massive de Russes pendant les années 1920 a en effet provoqué la première grande concertation internationale sur l'asile. Du côté russe, la Commission des juristes russes et arméniens a été la première à s'impliquer. En 1924, l'office central des réfugiés russes est devenu responsable de l'octroi du statut de réfugié aux exilés. Le Comité d'Émigration, également fondé en 1924 pour défendre les intérêts des Russes, rassemblait les associations communautaires et était chargé d'y coordonner les négociations. Le Zemgor a été créé en 1921 à la demande du gouvernement français pour prendre en charge l'assistance sociale aux réfugiés. Ces différentes institutions communautaires étaient des lieux de débats et d'expression de points de vue divergents, mais elles se rejoignaient dans la volonté d'œuvrer pour que les exilés obtiennent un statut de réfugié, pour qu'ils soient considérés dans leurs droits. Les anciens serviteurs de l'empire russe s'y sont investis, au bénéfice de l'ensemble de la communauté; c'est là un des caractères exceptionnels de cette immigration, que l'ouvrage de Catherine Gousseff met très clairement en lumière.

L'élaboration du statut de réfugié a pris une dizaine d'années. La définition du statut est passée par l'établissement d'un certificat d'identité par la Société des Nations en 1922, pour finalement être caractérisée par l'apatridie lors de la Convention de 1933. Ce statut était alors exempt de toute notion de persécution politique et/ou religieuse. En conséquence, les Russes, les Arméniens puis les Assyro-Chaldéens et les Turcs ont pu en bénéficier. Il n'a cependant pas été appliqué aux réfugiés venus d'Allemagne ou d'Italie au cours des années 1930 car ces derniers n'étaient pas nécessairement apatrides. L'auteure en conclut que la nouvelle législation a très vite généré de nouvelles exclusions. Le vide qu'entraînait une telle définition du réfugié n'a été compensé qu'avec la Convention de Genève de 1951. Selon Catherine Gousseff, l'écart entre les notions de réfugié par l'apatridie ou par la persécution (politique et/ou religieuse) explique que les analystes remontent rarement jusqu'à l'entre-deux guerres pour comprendre l'asile contemporain. Les mesures prises dans les années 1920 et 1930 étaient novatrices, mais étroitement liées à un contexte donné (volonté de régler définitivement les conséquences de la Première Guerre mondiale, opposition au régime soviétique...) et donc à des cas de figure précis. L'absence de réflexion théorique générale sur la condition d'exilé et de réfugié explique que le statut de réfugié de l'entre-deux guerres n'ait pu s'imposer de manière durable.

L'ouvrage, dense, est appuyé sur une recherche empirique très méticuleuse. L'auteur a mené un travail considérable d'exploitation d'archives, tirées notamment de l'OFPRA, de l'Office central des réfugiés russes à Paris et de la Société des Nations, mais aussi des archives de l'émigration russe conservées à Moscou, en Grande-Bretagne et aux ÉtatsUnis. L'ensemble est complété par trois enquêtes qualitatives menées en France et à 
Moscou, ainsi que par l'exploitation d'entretiens conduits par Claude Vernick auprès d'émigrés russes en France.

11 Après lecture de cette synthèse passionnante et foisonnante, l'immigration russe dans la France de l'entre-deux guerres est donc "normalisée ", délestée de nombreux stéréotypes et réinsérée dans l'histoire migratoire et le récit national français. De manière parallèle, Catherine Gousseff montre à quel point ce mouvement migratoire a concerné l'émergence de la problématique du réfugié. Ses élites ont contribué à son institutionnalisation par les États européens et les organismes internationaux spécialisés. L'exil russe ouvre de nombreuses pistes pour questionner le traitement contemporain de l'asile. Alors que les demandeurs d'asile en provenance de Russie (Tchétchénie, Daguestan, Ingouchie, etc.) sont parmi les plus nombreux dans l'espace européen (ils représentent le quatrième groupe en France pour le nombre de demandes, mais sont en seconde position pour la reconnaissance avec un taux de 12,8\% en $2009^{1}$ ), la quasi-absence de recherche scientifique consacrée à l'exil russe d'aujourd'hui ne permet pas de mettre en regard la situation contemporaine et celle de l'entre-deux guerres. L'absence d'implication d'organismes de représentation des populations migrantes dans les politiques et procédures d'attribution d'une protection apparaît comme une faiblesse du dispositif contemporain et marque ainsi l'éloignement des appareils décisionnels des besoins des exilés. Alors que l'Union européenne tente de mettre sur pied un dispositif européen de l'asile de plus en plus indexé sur les préoccupations sécuritaires des États membres et de reporter sur les États voisins une partie de ses responsabilités, il est important de revenir sur ce qui a autrefois poussé un nombre élevé d'États européens à s'impliquer de concert dans l'accueil des exilés russes. À l'heure où le Haut Commissariat aux Réfugiés développe une approche collective de l'asile au moyen de la politique de relocalisation (resettlement), c'est le principe fondamental du traitement individuel de la demande d'asile, formulé dès l'entre-deux guerres, qui est remis en cause.

\section{NOTES}

1. OFPRA (2009), Rapport d'activité 2009, Paris.

\section{AUTEURS}

\section{BÉNÉDICTE MICHALON}

Chargée de recherche CNRS, ADES (UMR 5185), Pessac 